\title{
A simple model for the total microbial biomass under occlusion of healthy human skin
}

\author{
H. Safuan $^{\text {a }}$, I. N. Towers $^{\text {a }}$, Z. Jovanoski ${ }^{\text {a }}$, H. S. Sidhu ${ }^{\text {a }}$ \\ ${ }^{a}$ Applied and Industrial Mathematics Research Group, School of Physical, Environmental and \\ Mathematical Sciences, University of New South Wales@ Canberra, Northcott Drive, Canberra ACT, \\ 2600 \\ Email: hamizah.mohdsafuan@student.adfa.edu.au
}

\begin{abstract}
Although human skin is a dry and acidic environment, there is an abundance of microflora that reside on its surface and are able to colonize it. If the skin environment is altered by an occlusive procedure, the quantity and composition of cutaneous bacteria changes drastically. Currently there are no mathematical models that attempt to describe these changes.

A non-autonomous logistic equation is used to model the total bacterial biomass during occlusion. The skin environment is represented as a time-dependent carrying capacity in the model. The changes of Carbon Dioxide Emission Rate which affect the microbial biomass has been integrated into the carrying capacity. The results have been compared with published experimental data and found to fit well.
\end{abstract}

Keywords: Skin occlusion, microbial biomass, non-autonomous logistic equation, variable carrying capacity 


\section{INTRODUCTION}

Human skin is a vital organ which has diverse functional properties. With multi-structured layers, it is the largest single organ of a human being. If the skin is stretched, it would cover an area of $1.75-1.8 \mathrm{~m}^{2}$ and weighs about $5 \mathrm{~kg}$ for an adult man. The main functions of the skin are to protect the human body from external injuries, serve as a barrier to infection or disease, regulate the body temperature and to sense stimulus from the environment. Three major layers of skin are the epidermis, dermis and hypodermis. The epidermis itself consists of layers and the only visible part is the outer most layer of the epidermis or stratum corneum (SC) (Alcamo and Krumhardt (2004)).

The common resident microflora on the skin surface are Propionibacterium, coagulase negative staphylococci (Staphylococci epidermidis), Malassezia, Micrococcus, aerobic coryneforms (lipophilic diphtheroids, nonlipophilic diphtheroids) and Acinetobacter. The transient microflora are Bacillus and coagulase positive staphylococci (Staphylococcus aureus) as listed by Bojar and Holland (2002). Fredricks (2001) mentioned the distribution of flora depends on the specific anatomical site, whether it is a dry, moist or oily area of the body. The axilla or toe web spaces can reach up to $10^{7}$ bacteria per $\mathrm{cm}^{2}$, whereas the forearm or trunk may harbour $10^{2}-10^{3}$ bacteria per $\mathrm{cm}^{2}$.

The normal skin environment is not a conducive one for microbes to colonise yet the resident bacteria live continuously on the skin. Despite its otherwise hostile nature to foreign lifeforms, the skin is capable of providing nutrients such as lipids and proteins to some microflora. In order to survive, some microbes will have to compete for their food and space with other microflora (Fredricks (2001)). Bacteria can adapt and react effectively instantaneously to the changes of the skin environment which can totally alter the normal microbial ecosystem.

Bojar and Holland (2002) discuss in detail the normal microflora of the skin and factors that control colonisation of it. Physiological factors such as hydration, $\mathrm{pH}$ level, oxygen and carbon dioxide emission, and the presence of enzymes, lipids and fatty acids determine the density and diversity of colonisation. In this paper, we model the total microbial biomass during occlusion taking into consideration some of these factors.

\section{SKIN OCCLUSION}

Occlusive treatments, such as the application of bandages to wounds, are ancient in origin but have only been the subject of dermatological experiments since the late 1960s. The goal of these experiments has been to investigate the significance of covering part of the skin with certain permeable or impermeable materials. As a therapy occlusions have been successfully applied to atopic dermatitis, psoriatic patients, and also to wounds, ulcers and cutaneous eruptions (Braham et al. (2010)).

Several experiments have sought to measure the physiological changes that take place on the surface of the skin whilst it is occluded for a period of time (Aly et al. (1978), Hartmann (1983) and Faergemann et al. (1983)). Aly et al. provides the most complete and comprehensive data, recording over 5 consecutive days the total microbial density, trans-epidermal water loss (TEWL), carbon dioxide emission rate (CDER) and skin-pH after a vinylidene polymer plastic film (Saran WrapC) was placed on the forearms of 10 healthy volunteers. Hartmann also studied the effect of a three day occlusion placed on the forearm of 26 volunteers whereas Faergemann et al. experimented with the effect of an 8 day skin occlusion on the back of 10 volunteers.

These experiments demonstrate when the skin is occluded the SC undergoes changes. Water from the deeper layers of the skin diffuse through the SC and is trapped by the occlusive dressing preventing the evaporation of water from the skin's surface. As the epidermal membrane becomes hydrated, the SC becomes more permeable which in turn results in an increase in the TEWL. In the study by Aly et al. the TEWL increased from $0.56 \mathrm{mg} / \mathrm{cm}^{2} / \mathrm{hr}$ to $1.87 \mathrm{mg} / \mathrm{cm}^{2} / \mathrm{hr}$. This increase in TEWL is evidence of increased skin-moisture content of the SC and skin surface. Hartmann found that the skin-moisture content increased from a low $20 \%$ and saturated at $75 \%$ after only three days of occlusion of the skin. According to the measurements of Faergemann et al. the TEWL reading remained relatively steady after the third day.

The skin also expels carbon dioxide to the atmosphere. Without any pressure on its surface, the skin 
releases carbon dioxide constantly at a low rate (Agache et al. (2004)). A hydrated and thus more permeable skin has a higher diffusion rate of carbon dioxide (CDER). In Aly et al. the CDER readings quickly rose to 3 times their base level by the first day and saturated there after.

Besides TEWL and CDER, changes in skin-pH also occurred during occlusion. The skin surface pH increased steadily from 4.38 to 7.05 . The occlusion inhibits the evaporation of eccrine perspiration thus elevating the $\mathrm{pH}$ value. Faergemann et al. also found the $\mathrm{pH}$ to rise rapidly during the first three days and remained fairly constant until the 8th day. Other factors that might be important to the $\mathrm{pH}$ change are the changes to the composition of sweat, sebum, lipids, fatty acids and nutrients (Schmid and Korting (2006)) during the occlusion. These latter quantities have not been measured because they are complex and not easily interpreted.

Under an occlusion, the normally harsh skin environment is transformed to one more favourable with enriched nutrient flows resulting in an increase in the density of micro-organisms found there. Aly et al. measured an almost 4 orders of magnitude increase in the total microbial density in the first two days after the application of the occlusion and it remained approximately constant for the remaining three days of the experiment. Hartmann (1983) found similar rises in the microbial density after a three day occlusion of the skin, but Faergemann et al. reported only two orders of magnitude increase for microbes residing on the back.

We are interested in modelling the growth of the total microbial biomass under an occlusion. Since all of the physiological factors impact upon bacterial counts, our mathematical model attempts to incorporate one or more of these factors.

\section{LOGISTIC EQUATION TO MODEL MICROBIAL BIOMASS}

In the pre-occluded skin, the environment is relatively constant and the microbes receive a continuous source of nutrients. The density of microbes is in equilibrium with its environment. A common model to describe such a population is the Verhulst equation or the logistic equation (Murray (1989))

$$
\frac{d N(t)}{d t}=r N(t)\left[1-\frac{N(t)}{K}\right], \quad N(0)=N_{0}
$$

where $N(t)$ denotes the population density, $r$ is the intrinsic growth rate, $K$ is the carrying capacity, $t$ is time and $N_{0}$ is the initial population density at time $t=0$. It is an autonomous differential equation since only $N(t)$ is time-dependent whereas $r$ and $K$ are positive constants. The solution of (1) can be obtained by separation of variables

$$
N(t)=\frac{K N_{0}}{K e^{-r t}+N_{0}\left(1-e^{-r t}\right)} .
$$

Equation (1) has been widely used because of its simplicity, existence of an exact solution and straightforward stability analysis. According to the logistic equation any initial population will grow until reaching the maximum sustainable level; the carrying capacity $K$. In the pre-occluded skin $K \approx N_{0}=920$ counts $/ \mathrm{cm}^{2}$.

When an occlusion is applied to the skin the environment beneath it begins to change to one that is generally more favourable for microbial growth. A changing environment may be characterised by a varying carrying capacity and possibly a varying intrinsic growth rate. The appropriate way to model such a scenario is the non-autonomous logistic equation

$$
\frac{d N(t)}{d t}=r(t) N(t)\left[1-\frac{N(t)}{K(t)}\right] .
$$

The general mathematical properties of (3) were deduced by Coleman (1979) and later modified by Hallam and Clark (1981) to consider population growth in a polluted environment. Exact solutions exist only for a limited number of cases (Banks (1994)).

In what follows we assume the intrinsic growth rate, $r$, is constant and seek a monotonically increasing function, $K(t)$, that begins with carrying capacity $K(0)=K_{0}, K_{0} \approx N_{0}$, representing the unoccluded 
skin with baseline values for TEWL, CDER, skin-moisture, skin-pH and nutrient flow rates to a new and larger carrying capacity, $K_{s}$, representing an environment with elevated but constant values for the aforementioned physiological factors. Meyer (1994) and Meyer and Ausubel (1999) introduced a logistic form of the carrying capacity but a much simpler model that meets our criteria is expressed as

$$
\frac{d K}{d t}=-\alpha\left(K-K_{s}\right)
$$

where $\alpha$ is the saturation constant. The solution for the carrying capacity is

$$
K(t)=K_{s}\left(1-\beta e^{-\alpha t}\right),
$$

where $\beta=1-K_{0} / K_{s}$. A carrying capacity of this form has previously been used to model fish stocks in a nutrient enriched environment (Ikeda and Yokoi (1980)).

It is possible to use the data of the microbial densities to estimate $r, K_{0}$ and $K_{s}$ but not the value of $\alpha$. For this purpose we take a different approach. From the discussion of the previous section, the CDER and TEWL co-evolve with the environment. This means that the changes in the carrying capacity correlate positively with the changes of both CDER and TEWL. Therefore, we assume that the functional form for both of these quantities are given by expressions similar to that of equation (5), with the same parameter $\alpha$.

Here we use the CDER data taken from Aly et al.. Let $C(t)$ be the CDER at time $t$, then the time evolution is given by $C(t)=C_{s}\left(1-\beta_{c} e^{-\alpha t}\right)$ where $\beta_{c}=1-C_{0} / C_{s}$. This requires the estimation of two further parameters, $C_{0}$ and $C_{s}$. To be clear, we use the CDER data to estimate the value of $\alpha$ which in turn is used in the expression for $K(t)$.

\section{RESULTS AND VALIDATION}

The CDER and total microbial density data from Aly et al. (1978) were extracted using G3DATA (Frantz (2011)) and are reproduced in Figure 1 and Figure 2a, respectively. There are some inconsistencies between the graphs and the text in Aly et al.. Throughout this discussion we will assume that the data from the graphs is correct while the text is in error.

Using the CDER observations of Figure 1 , we set $C_{0}=25 \mathrm{nl} / \mathrm{cm}^{2} / \mathrm{min}$ and estimate $C_{s} \approx$ $91.2 \mathrm{nl} / \mathrm{cm}^{2} / \mathrm{min}$, it being the average of the observations over the last three days of the occlusion. A nonlinear least squares fit was then used on the CDER data to estimate $\alpha$. We obtained $\alpha=1.92 /$ day with a $95 \%$ confidence interval of $(0.29,3.54)$. The large confidence interval is a consequence of the small number of observations. The fitted curve is shown in Figure 1. The model appears to adequately represent the observed data.

We now turn our attention to the microbial density data. Since we are dealing with a general biomass and not a specific bacterium and that in vivo bacterial growth rates are not known, we must estimate $r$ for the data of Figure 2a.

The intrinsic growth rate, $r \approx \Delta \ln N / \Delta t$, can be determined from the so-called exponential growth phase of the growth curve. By taking the average slope from the first day and second day relative to the pre-occlusion microbial density we get $r=6.58 /$ day. Further, we set $K_{0}=920 \mathrm{counts} / \mathrm{cm}^{2}$, and estimate $K_{s}=2.29 \times 10^{7}$ counts $/ \mathrm{cm}^{2}$, it being the geometric mean of the microbial density over the last three days. No attempt was made to find standard errors for these estimates due to the small size of the data set.

Incorporating equation (5) into equation (3) leads to

$$
\frac{d N(t)}{d t}=r N(t)\left[1-\frac{N(t)}{K_{s}\left(1-\beta e^{-\alpha t}\right)}\right] .
$$

Numerical solution of equation (6) with the estimated parameters is shown in Figure 2a. It is apparent that the model does a good job in describing the growth of the microbial biomass. What is also clear is that this model predicts the very rapid increase in the environment's carrying capacity. 


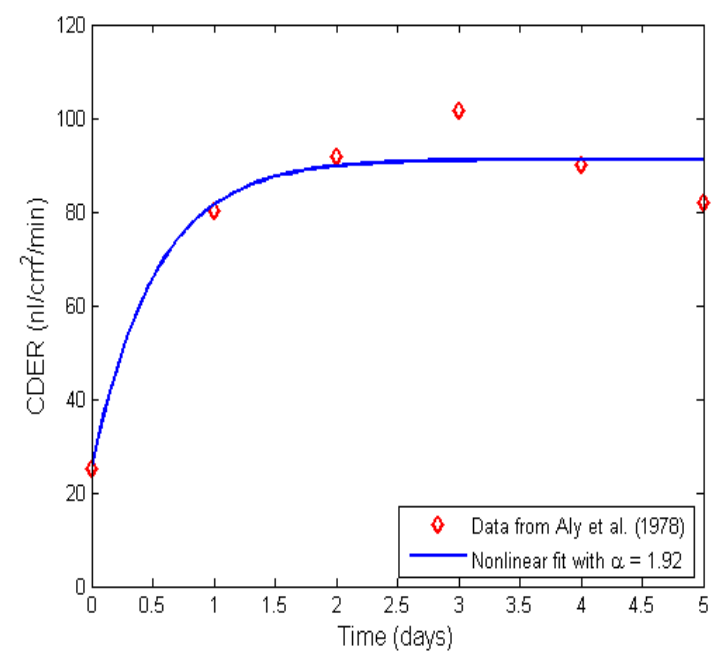

Figure 1: CDER data with the nonlinear fitted curve.

The ability to validate a model with an independent set of data is vitally important. To validate our model we use the data of Pillsbury and Kligman (1967) (report released by US Army in 2000). Pillsbury and Kligman reported the total microbial density on forearm skin under occlusion for a period of more than 2 weeks. The data is reproduced in Figure 2b. As with other experiments mentioned so far, a four orders of magnitude increase of cutaneous microflora resulted within the two days of the application of an occlusion. The geometric mean was calculated for each of the days for which the microbial density was measured. Using the same model with the same parameters as for the Aly et al., our model predicts the total microbial biomass remarkably well.

\section{DISCUSSION AND CONCLUSION}

The carrying capacity, $K(t)$ in the model (6) encompasses the adjustment of the environment resulting from the changes on the skin surface due to the application of an occlusion.

From the model we deduce that there exist two characteristic time lengths at play. One related to the increase in the carrying capacity and the other to the increase of the population density. From equation (4) the quantity $\left|K(t)-K_{s}\right|$ represents the environment's instantaneous excess capacity and the time required to halve this capacity is $t_{1 / 2}=\ln 2 / \alpha \approx 8.7$ hours. Whereas, the doubling time for the microbial biomass is $t_{1 / 2}=\ln 2 / r \approx 2.5$ hours. This suggests that by the first 8.7 hours after the occlusion is placed on the skin the excess capacity is decreased (carrying capacity increased) by approximately $K_{s} / 2 \approx 10^{7}$ counts $/ \mathrm{cm}^{2}$. In the meantime the microbial density has experienced only a 10-fold increase. Initially, the environment under the occlusion is developing at an extremely fast rate enabling the growth of the total skin microflora without restraint. This feature of the model is evident from Figures $2 \mathrm{a}$ and $2 \mathrm{~b}$.

There are several limitations of this study. The model possibly could be improved if we acquire a greater number of datasets that observed a set of physiological effects for a longer period of occlusion time. Better quality CDER data, for example, would provide a better estimate for $\alpha$. Of course, it might be possible to use the TEWL data to find another independent estimate of $\alpha$. However, this was not done here because of a problem with the quality of the data in Aly et al.. The TEWL was measured about 20 minutes after the occlusion was removed. Recently Pinto and Rodrigues (2005) recorded the TEWL immediately after the removal of a one-day occlusion of the forearm for 30 minutes. It was found that the TEWL fell to less than half its maximum level within a 20 minute interval. This demonstrates that the TEWL data in Aly et al. grossly underestimates the true value, at least for the first and perhaps the second day, and any estimate of $\alpha$ based on this data would also have been an underestimate. 


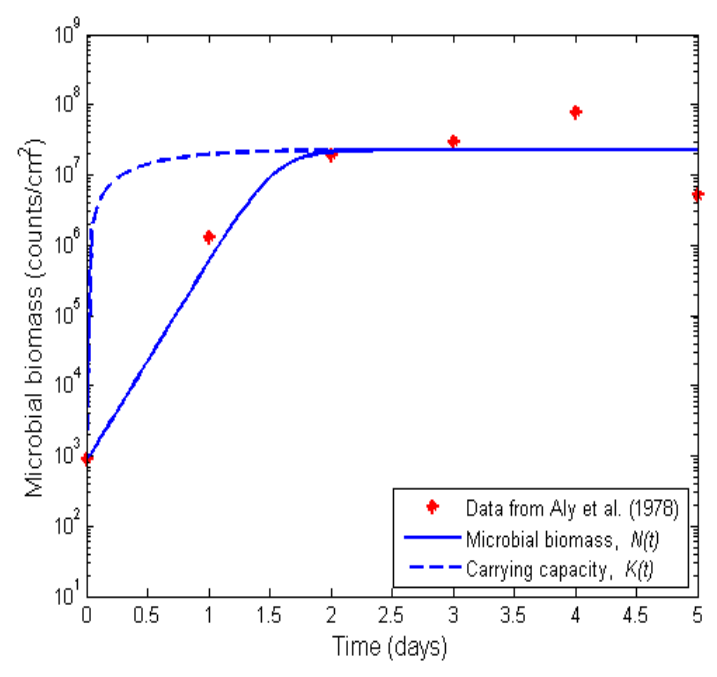

(a)

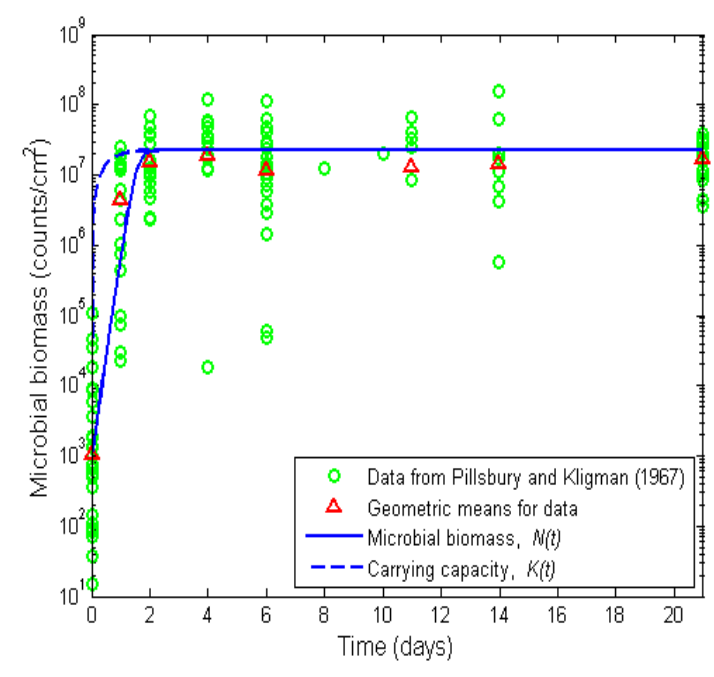

(b)

Figure 2: Growth curves $N(t)$ and variable carrying capacity $K(t)$. (a) Aly et al. (1978) data with the estimated parameters (b) Pillsbury and Kligman (1967) data using the proposed model.

In experiments, the intrinsic growth rate depends on temperature, water content, $\mathrm{pH}$ and $\mathrm{CO}_{2}$, to mention a few (Denyer et al. (1990), Ratkowsky and Ross (1995)). Our assumption of a constant $r$ is certainly not valid, but how the effects of an occlusion is reflected in an increase in $r$ is not possible to ascertain. Furthermore, we a dealing with a biomass consisting of four different species of bacteria and for each in vivo growth rates are not known.

Future work will need to focus on the interaction between the dominant bacterial species present. The work of Aly et al. and Pillsbury and Kligman (1967) present an interesting dynamic between the major bacterial species of the skin. A better understand of these interactions could assist in building a model of the time evolution of bacterial growth in a wound, with the obvious health implications.

In summary, we have used a simple logistic model with varying carrying capacity to describe the growth of a microbial biomass under an occlusive patch on the skin. The model provides a remarkably good fit to existing data. Our model is a first attempt to mathematically describe bacterial growth under an occlusion and we hope to develop it further for possible use of occlusions placed on wounded skin.

\section{ACKNOWLEDGEMENT}

The first author would like to thank Universiti Tun Hussein Onn Malaysia for the postgraduate scholarship. Financial support from UNSW Canberra is greatly appreciated.

\section{REFERENCES}

Agache, P. G., P. H. P. Agache, and H. I. Maibach (2004). Skin Structural Components:Physiology and Metrology, In:Measuring the skin, pp. 194. Springer, Germany.

Alcamo, I. E. and B. Krumhardt (2004). The Integumentary System, In:Anatomy and Physiology the Easy Way, pp. 93-97. Barron's Educational Series, New York.

Aly, R., C. Shirley, B. Cunico, and H. I. Maibach (1978). Effect of prolonged occlusion on the microbial flora, ph, carbon dioxide and transepidermal water loss on human skin. Journal of Investigative Dermatology 71, 378-381. 
H. Safuan et al., A simple model for the total microbial biomass under occlusion of healthy human skin

Banks, R. B. (1994). Phenomena with Variable Carrying Capacity, In:Growth and diffusion phenomena:Mathematical frameworks and applications, pp. 241-285. Springer-Verlag, USA.

Bojar, R. A. and K. T. Holland (2002). Review: the human cutaneous microflora and factors controlling colonisation. World Journal of Microbiology \& Biotechnology 18, 889-903.

Braham, S. J., R. Pugashetti, and H. I. Maibach (2010). Occlusive therapy in atopic dermatitis: Overview. Journal of Dermatological Treatment 21, 62-72.

Coleman, B. D. (1979). Nonautonomous logistic equations as models of the adjustment of populations to environmental change. Mathematical Biosciences 45, 159-173.

Denyer, S. P., M. C. Davies, J. A. Evans, R. G. Finch, D. G. E. Smith, M. H. Wilcox, and P. Williams (1990). Influence of carbon dioxide on the surface characteristics and adherence potential of coagulasenegative staphylococci. Journal of Clinical Microbiology 28, 1813-1817.

Faergemann, J., R. Aly, D. R. Wilson, and H. I. Maibach (1983). Skin occlusion:effect on pityrosporum orbiculare, skin ph, transepidermal water loss, and water content. Archives of Dermatological Research 275, 383-387.

Frantz, J. (2000 (accessed March 20, 2011)). G3DATA. http://www. frantz.fi/software/ g3data.php.

Fredricks, D. N. (2001). Microbial ecology of human skin in health and disease. Journal of Investigative Dermatology Symposium Proceedings 6, 167-169.

Hallam, T. G. and C. E. Clark (1981). Non-autonomous logistic equations as models of populations in a deteriorating environment. Journal of Theoretical Biology 93, 303-311.

Hartmann, A. A. (1983). Effect of occlusion on resident flora, skin moisture and skin ph. Archives of Dermatological Research 275, 251-254.

Ikeda, S. and T. Yokoi (1980). Fish population dynamics under nutrient enrichment - a case of the east seto inland sea. Ecological Modelling 10, 141-165.

Meyer, P. (1994). Bi-logistic growth. Technological Forecasting and Social Change 47, 89-102.

Meyer, P. and J. H. Ausubel (1999). Carrying capacity:a model with logistically varying limits. Technological Forecasting and Social Change 61, 209-214.

Murray, J. D. (1989). Continuous Population Models for Single Species, In:Mathematical Biology, pp. 2-3. Springer-Verlag, USA.

Pillsbury, D. M. and A. M. Kligman (1967). Chronic disabling dermatoses. Technical report, U. S. Army Medical Research and Development Command.

Pinto, P. C. and L. M. Rodrigues (2005). Influence of the time of occlusion on the quantitative parameters obtained by modelling trans-epidermal water loss curves to determine the human cutaneous barrier function in vivo. Medical \& Biological Engineering \& Computing 43, 771-775.

Ratkowsky, D. A. and L. Ross (1995). Modelling the bacterial growth/no growth interface. Letters in Applied Microbiology 20, 29-33.

Schmid, M. H. and H. C. Korting (2006). The ph of the skin surface and its impact on the barrier function. Skin Pharmacology and Physiology 19, 296-302. 